\title{
Coenzyme Q10 alleviates sevoflurane-induced neuroinflammation by regulating the levels of apolipoprotein $E$ and phosphorylated tau protein in mouse hippocampal neurons
}

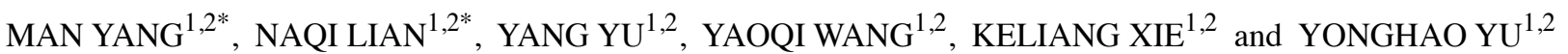 \\ ${ }^{1}$ Department of Anesthesia, ${ }^{2}$ Tianjin Institute of Anesthesiology, \\ Tianjin Medical University General Hospital, Tianjin 300052, P.R. China
}

Received November 17, 2019; Accepted March 30, 2020

DOI: $10.3892 / \mathrm{mmr} .2020 .11131$

\begin{abstract}
Sevoflurane may exert neurotoxic effects on the developing brain. Coenzyme Q10 (CoQ10) has been reported to reduce sevoflurane anesthesia-induced cognitive deficiency in 6-day-old mice. However, its specific mechanisms remain unknown. Apolipoprotein $\mathrm{E}$ (ApoE) has been reported to lead to the initiation of neurodegeneration in patients with Alzheimer's disease (AD) and may serve an important role in anesthesia-induced neurotoxicity. The present study aimed to reveal the role of ApoE in the pathogenesis of tau protein hyperphosphorylation and neuroinflammation enhancement caused by sevoflurane anesthesia, as well as the protective mechanism of CoQ10 in an anesthetic sevoflurane treatment model of primary mouse hippocampal neurons. For that purpose, the neurons were randomly assigned to the following groups: i) Control; ii) sevoflurane; iii) control+corn oil; iv) sevoflurane+corn oil; v) control+CoQ10; and vi) control+CoQ10. CoQ10 or corn oil alone was added to the medium on day 4 of neuron culture. The neurons in the sevoflurane group were treated with $21 \% \mathrm{O}_{2}, 5 \% \mathrm{CO}_{2}$ and $4.1 \%$ sevoflurane for $4 \mathrm{~h}$, whereas the control group only with $21 \%$ $\mathrm{O}_{2}$ and $5 \% \mathrm{CO}_{2}$ on day 5. Samples were collected immediately after anesthesia or control treatment. ATP, superoxidase dismutase (SOD)1, ApoE mRNA, total ApoE, full-length ApoE, ApoE fragments, Tau5, Tau-PS202/PT205 (AT8), Tau-PSer396/404 (PHF1), tumor necrosis factor (TNF)- $\alpha$, interleukin (IL)-6 and IL-1 $\beta$ levels were measured with ELISA, quantitative PCR, western blotting and immunocytochemistry.
\end{abstract}

Correspondence to: Dr Yang Yu or Professor Yonghao Yu, Department of Anesthesia, Tianjin Medical University General Hospital, 154 Anshan Road, Tianjin 300052, P.R. China

E-mail: sevenyu1987@outlook.com

E-mail: yuyonghao@126.com

*Contributed equally

Key words: sevoflurane, ApoE, ApoE fragments, tau phosphorylation, primary neuron, coenzyme Q10
The results of the present study indicated that sevoflurane anesthesia significantly decreased the ATP and SOD levels, but increased ApoE mRNA, total ApoE protein, full-length ApoE, ApoE fragments, phosphorylated tau (AT8 and PHF1) and neuroinflammatory factor (TNF- $\alpha$, IL-6 and IL-1 $\beta$ ) expression levels compared with those in the control group. The use of CoQ10 reversed the expression of these factors. These results suggested that sevoflurane treatment damaged mouse hippocampal neurons, which may be associated with the expression of ApoE and its toxic fragments. CoQ10 improved energy replenishment and inhibited oxidative stress, which may lead to a decrease in ApoE and phosphorylated tau protein expression, thus mitigating the sevoflurane-induced neuroinflammation in mouse hippocampal neurons.

\section{Introduction}

Sevoflurane is the most commonly used inhalant anesthetic in pediatric surgery (1); however, it exerts neurotoxic effects on the developing brain (2). Clinical studies have reported that children with long-time or multiple exposures to general anesthesia and surgery before 3-4 years of age may have an increased risk of learning and memory disability $(1,3)$. In addition, numerous studies have reported that the use of inhalant anesthetics such as sevoflurane can cause cognitive impairment, brain structural changes, neuroinflammation, apoptosis and synaptic deficits in animal or cell models (4-8). Since millions of neonates and children worldwide require surgery under anesthesia each year (9), exploring the mechanisms underlying sevoflurane-induced neurotoxicity in the developing brain and finding an approach to alleviate the postoperative neurocognitive disorder in children are not only of general scientific interest, but may also have substantial impact on public health.

Apolipoprotein E (ApoE), a 35-kDa glycoprotein, is a type of lipoprotein component important in controlling lipoprotein metabolism and cholesterol homeostasis (10). Previous studies have suggested that ApoE may serve a protective role in neuronal activity and injury repair in the central nervous system $(10,11)$. However, specific neurotoxic ApoE fragments, such as an $18 \mathrm{kDa}$ N-terminal fragment, have been reported to be directly associated with the formation of amyloid- $\beta$ plaques, 
tau hyperphosphorylation, neurofibrillary triangle formation, and the initiation of neuroinflammation and neurodegeneration in patients with Alzheimer's disease (AD) (11-13). In addition, several studies have also demonstrated that tau protein hyperphosphorylation and neuroinflammation enhancement may be the main reason for anesthesia-induced cognitive impairment in young mice $(5,6,14)$. However, whether ApoE and its fragments contribute to tau phosphorylation and neuroinflammation following general anesthesia remains to be investigated.

Coenzyme Q10 (CoQ10), a strong antioxidant and mitochondrial energy storage agent, has neuroprotective, energy conversion, anti-inflammatory and anti-oxidative effects $(15,16)$. $\mathrm{Xu}$ et al (17) reported that an intraperitoneal injection of CoQ10 prior to anesthesia reduced sevoflurane-induced mitochondrial dysfunction and cognitive deficiency in 6-day-old mice. However, the specific underlying mechanism remains unknown.

The present study aimed to reveal the role of ApoE in the pathogenesis of tau protein hyperphosphorylation and neuroinflammation induced by sevoflurane anesthesia, as well as the protective mechanism of CoQ10 in an anesthetic sevoflurane treatment model of primary mouse hippocampal neurons.

\section{Materials and methods}

Primary neuron culture with serum free medium and treatment. All experimental protocols in the present study were approved by the Animal Experimental Ethics Committee of Tianjin Medical University General Hospital (Tianjin, China; approval no. 2018-X6-11). Pregnant C57BL/6J mice (2-month old, weight 20-25 g, n=5) were purchased from Beijing Huafukang Biotechnology Co., Ltd. and were housed under a 12-h light/dark cycle with food and water provided ad libitum. The room temperature was $22-24^{\circ} \mathrm{C}$ and the humidity $40-50 \%$. The mice were decapitated on gestation day 15 , and the embryos were removed and soaked in $75 \%$ ethanol for $1 \mathrm{~min}$. The hippocampal tissue of the fetal mice was removed intact, repeatedly cut into fragments with scissors and transferred to a petri dish containing DMEM (Gibco; Thermo Fisher Scientific, Inc.). Next, $0.25 \%$ trypsin was added to the petri dish for digestion in a $37^{\circ} \mathrm{C}$ thermostat for $30 \mathrm{~min}$. The tissue fragments and digestive juices were transferred to a centrifuge tube, and an equivalent volume of digestive stop fluid (DMEM+10\% FBS; Gibco; Thermo Fisher Scientific, Inc.) was added and mixed gently for $15 \mathrm{~min}$. Subsequently, the mixture was strained through a $60-\mu \mathrm{m}$ sieve. After $1 \mathrm{~min}$, the supernatant containing a single-cell suspension was collected and mixed with a digestive stop fluid (DMEM+10\% FBS; Gibco; Thermo Fisher Scientific, Inc.). Cells were counted under a microscope to ensure a density of $7.0 \times 10^{6}$ cells $/ \mathrm{ml}$. Then, $1.5 \mathrm{ml}$ of cell and digestive stop fluid mixture was inoculated into each well of a 6-well plate. After $4 \mathrm{~h}$ of culture, neuron elongation was observed using serum free medium (Neurobasal-A+2\% B27; Engreen Biosystem Ltd.) in place of digestive stop fluid. Neuron culture medium was replaced every 3 days.

Neurons cultured for 5 days were used in the experiments. The neurons were randomly assigned into the following groups: i) Control; ii) sevoflurane; iii) control+corn oil (the vehicle of CoQ10); iv) sevoflurane+corn oil; v) control+CoQ10; and vi) control+CoQ10. Each dish of neurons in the corn oil groups was incubated with neuron culture medium mixed with $500 \mu 1$ corn oil overnight on day 4 of cell culture. For neurons in the CoQ10 group, $500 \mu \mathrm{g}$ CoQ10 (dissolved in corn oil at $1.5 \mathrm{mg} / \mathrm{ml}$ ) was added to each dish on day 4 and incubated overnight at $37^{\circ} \mathrm{C}$. Neurons in the sevoflurane group were placed in a sealed resin box $(20 \times 15 \times 7 \mathrm{~cm})$ and treated with $21 \% \mathrm{O}_{2}, 5 \% \mathrm{CO}_{2}$ and $4.1 \%$ sevoflurane (2X minimum alveolar concentration) for $4 \mathrm{~h}$ in a $37^{\circ} \mathrm{C}$ incubator as described by $\mathrm{Lu}$ et al (5). The control groups were treated with $21 \% \mathrm{O}_{2}$ and $5 \% \mathrm{CO}_{2}$ for $4 \mathrm{~h}$ under the same conditions. A variable anesthetic gas monitor (Vamos; Dräger Medical AG \& Co. KgaA) was used to continuously monitor the concentration of $\mathrm{O}_{2}, \mathrm{CO}_{2}$ and sevoflurane during anesthesia or control treatment.

Cell Counting Kit-8 (CCK-8) cell viability assay. CCK-8 was used according to the manufacturer's protocol. When sevoflurane or control treatment was finished, the original culture medium in each well was replaced with $90 \mu 1$ serum free medium and $10 \mu \mathrm{l} \mathrm{CCK8} \mathrm{reagent} \mathrm{(cat.} \mathrm{no.} \mathrm{CK04;} \mathrm{Dojindo}$ Molecular Technologies, Inc.) was added immediately, followed by incubation at $37^{\circ} \mathrm{C}$ in the dark for $2 \mathrm{~h}$. The absorbance value at $450 \mathrm{~nm}$ was measured with a VERSAMax microplate reader (Molecular Devices, LLC) to determine the cell viability. The viability of the control group was considered as $100 \%$, and the results of the other groups were calculated as the percentage of the control group.

Primary neuron harvesting. The neurons in different groups were collected for biochemical tests immediately after anesthesia or control treatment. The culture medium was removed, and the petri dishes were washed three times with PBS. Then, $1 \mathrm{ml}$ RIPA buffer (Beijing Solarbio Science \& Technology Co., Ltd.) was added, and the neurons were scraped out carefully from the bottom of the petri dish with a cell curette. Finally, the lysates were collected and then centrifuged at $12,000 \mathrm{x} \mathrm{g}$ for $10 \mathrm{~min}$ at $4^{\circ} \mathrm{C}$.

Reverse transcription-quantitative PCR (RT-qPCR). Total RNA extraction was performed by the RNA TRIzol ${ }^{\circledR}$ isolation reagent kit (Invitrogen; Thermo Fisher Scientific, Inc.). Total RNA concentration was determined using a spectrophotometer. A total of $500 \mathrm{ng}$ mRNA in each group was reverse transcribed into cDNA with the All-in-One First-Strand cDNA Synthesis kit (cat. no. AORT-0050; GeneCopoeia, Inc.), and amplified by PCR with SYBR ${ }^{\circledR}$ Green qPCR Master Mix (MedChemExpress LLC.) using an $\mathrm{iQ}^{\mathrm{TM}} 5$ system (Bio-Rad Laboratories, Inc.) under the following conditions: $5 \mathrm{~min}$ at $95^{\circ} \mathrm{C}$ and 40 cycles of $15 \mathrm{~s}$ at $95^{\circ} \mathrm{C}$ and $60^{\circ} \mathrm{C}$ for $1 \mathrm{~min}$. Relative mRNA levels were calculated by the $2^{-\Delta \Delta \mathrm{Cq}}$ method (18). The primer sequences were as follows: GAPDH forward, 5'-TGT AGACCATGTAGTTGAGGTCA-3' and reverse, 5'-AGGTCG GTGTGAACGGATTTG-3'; and ApoE forward, 5'-GACCCA GCAAATACGCCTG-3' and reverse, 5'-CATGTCTTCCAC TATTGGCTCG-3'.

ELISA. The levels of total ApoE, inflammatory factors interleukin (IL)-1 $\beta$, IL-6 and tumor necrosis factor (TNF)- $\alpha$, superoxidase dismutase (SOD)1 and ATPase in the neurons of each group were determined using mouse ELISA kits (ApoE, cat.no. E-EL-M0135; 

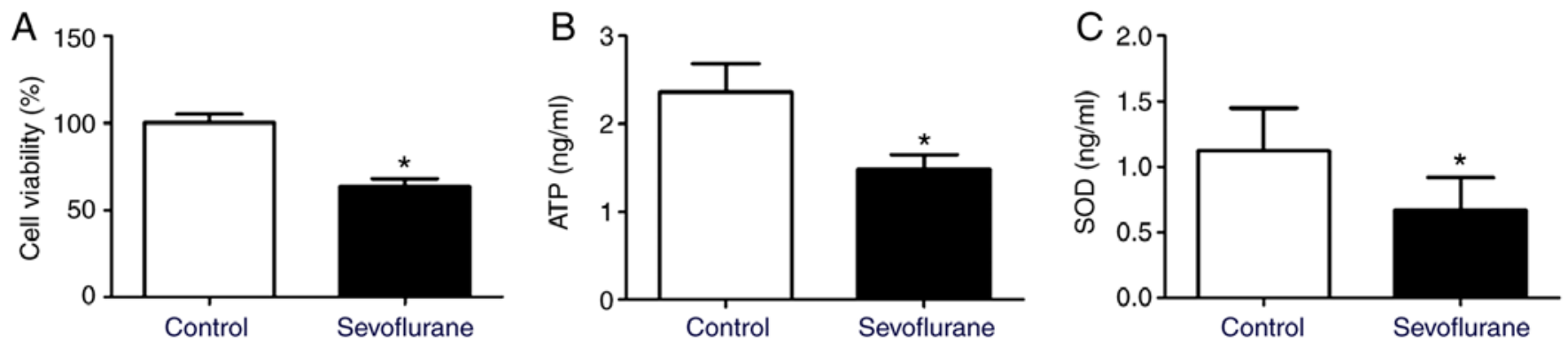

Figure 1. Effects of sevoflurane anesthesia on cell viability, ATP levels and SOD contents in mouse hippocampal neurons. (A-C) Neurons were harvested after sevoflurane or control treatment; (A) Cell Counting Kit-8 assay was used to examine cell viability and ELISA was used to test the contents of (B) ATP and (C) SOD in mouse hippocampal neurons. ${ }^{*} \mathrm{P}<0.05$. SOD, superoxide dismutase.

IL1- $\beta$, cat. no. E-EL-M0037c; IL-6, cat. no. E-EL-M0044c; TNF- $\alpha$, cat. no. E-EL-M0049c; SOD1, cat. no. E-EL-M2398c; and ATPase, cat. no. E-EL-M0813c; all from Elabscience Biotechnology, Inc.). Briefly, $100 \mu \mathrm{l}$ standard or neuron protein was added to each well and incubated at $37^{\circ} \mathrm{C}$ for $90 \mathrm{~min}$. Then, $100 \mu \mathrm{l}$ specific antibody was added to each well and incubated at $37^{\circ} \mathrm{C}$ for an additional $60 \mathrm{~min}$. Subsequently, $100 \mu 1$ enzyme conjugate was added to each well and incubated at $37^{\circ} \mathrm{C}$ for $30 \mathrm{~min}$. After five washes, $90 \mu \mathrm{l}$ substrate solution was added for $15 \mathrm{~min}$, followed by $50 \mu \mathrm{l}$ terminating solution to stop the reaction. Finally, a fluorescence reader was used to measure the optical density (OD) of each well at $450 \mathrm{~nm}$.

Western blot analysis. Western blotting was used to detect the expression of full-length ApoE, ApoE fragments, Tau5, AT8 and PHF1 in neurons. Total protein was quantified using the a bicinchoninic acid protein assay kit (Pierce; Thermo Fisher Scientific, Inc.).

Neuron protein $(50 \mu \mathrm{g})$ per well was separated by $4-20 \%$ SDS-PAGEand transferred toa nitrocellulosemembrane(Bio-Rad Laboratories, Inc.). The membrane was blocked in 5\% skimmed milk powder in TBS for $2 \mathrm{~h}$ at $37^{\circ} \mathrm{C}$ and incubated overnight at $4^{\circ} \mathrm{C}$ with primary antibodies against the following proteins: Full-length ApoE and ApoE fragments (1:4,000; cat. no. 178479; EMD/Merck KGaA), Tau5 (1:1,000; cat. no. ABN454; EMD Millipore), AT8 (1:2,000; cat. no. MN1020; Thermo Fisher Scientific, Inc.), PHF1 (1:1,000; cat. no. 3Ab184951; Abcam) and $\beta$-actin (1:2,000; cat. no. A5060; Sigma-Aldrich; Merck $\mathrm{KGaA})$. After rinsing with TBS, a goat anti-mouse $\operatorname{IgG}(\mathrm{H}+\mathrm{L})$ secondary antibody (1:5,000; cat. no. 31430; Invitrogen; Thermo Fisher Scientific, Inc.) was added and incubated at $37^{\circ} \mathrm{C}$ for $1 \mathrm{~h}$. The membrane was washed in TBS+0.2\% Tween-20 prior to adding the ECL reagent (cat. no. 34577; Invitrogen; Thermo Fisher Scientific, Inc.). Then, the membrane was scanned and photographed using a quantitative Gel Quantity One system (FluorChem FC3, ProteinSimple Inc.). The expression level of the target protein was calculated as the ratio of the integral optical density of the target band to the $\beta$-actin band. For comparison across experimental conditions, $100 \%$ of protein level changes refer to the control levels for the purpose of comparison to the experimental conditions.

Immunocytochemistry. Slides $(2 \times 2 \mathrm{~cm})$ were pre-coated in the culture dish prior to neuron culture. Following sevoflurane or control treatment, the culture medium in the dishes was removed, and the dishes were washed three times with pre-cooled PBS. Each well was fixed with $1 \mathrm{ml} 4 \%$ paraformaldehyde for $30 \mathrm{~min}$ at $37^{\circ} \mathrm{C}$, washed five times and treated with $0.3 \%$ Triton for $30 \mathrm{~min}$ at $37^{\circ} \mathrm{C}$. The neurons were blocked for $1 \mathrm{~h}$ in $10 \%$ goat serum (Gibco; Thermo Fisher Scientific, Inc.) at $37^{\circ} \mathrm{C}$. After removing any excess liquid, anti-AT8 (1:1,000; cat. no. MN1020; Thermo Fisher Scientific, Inc.) and anti-MAP2 (1:1,000; cat. no. EPR19691; Abcam) antibodies were added to each well. The slides were placed in a wet box with PBS and incubated overnight at $4^{\circ} \mathrm{C}$. After washing three times with PBS, Alexa Fluor ${ }^{\circledR} 488$ goat anti-mouse IgG (1:500; cat. no. A-11001; Thermo Fisher Scientific, Inc.) and Alexa Fluor $^{\circledR} 594$ goat anti-rabbit IgG (1:500; cat. no. A-11012; Thermo Fisher Scientific, Inc.) secondary antibodies were added to the slides and incubated for $1 \mathrm{~h}$ at room temperature in the dark. After washing the slides three times with PBS, the slides were incubated with DAPI (cat. no. 104139; Abcam) in a humidified dark chamber for $10 \mathrm{~min}$ at $37^{\circ} \mathrm{C}$ and subsequently washed five times. Images (magnification, $\mathrm{x} 40$ ) were observed and imaged captured under a fluorescence microscope.

Statistical analysis. The data are presented as the mean \pm standard deviation. The number of samples was four per group in the RT-qPCR, western blotting, ELISA and immunohistochemistry assays. Statistical analysis was performed using GraphPad Prism software version 5.0 (GraphPad Software, Inc.) and SPSS statistical software version 21.0 (IBM Corp.). Unpaired Student's t-test (if the values followed a Gaussian distribution) or Mann-Whitney test (if the values did not follow a Gaussian distribution) was used to analyze the difference between two groups in the biochemistry data. $\mathrm{P}<0.05$ was considered to indicate a statistically significant difference.

\section{Results}

Sevoflurane anesthesia reduces cell viability, ATP level and SOD contents in mouse hippocampal neurons. Compared with the control group, the levels of ATP and SOD, as well as the cell viability decreased significantly in neurons after sevoflurane treatment (Fig. 1).

Sevoflurane anesthesia increases the expression of ApoE and its toxic fragments in mouse hippocampal neurons. The western blotting results revealed that compared with 


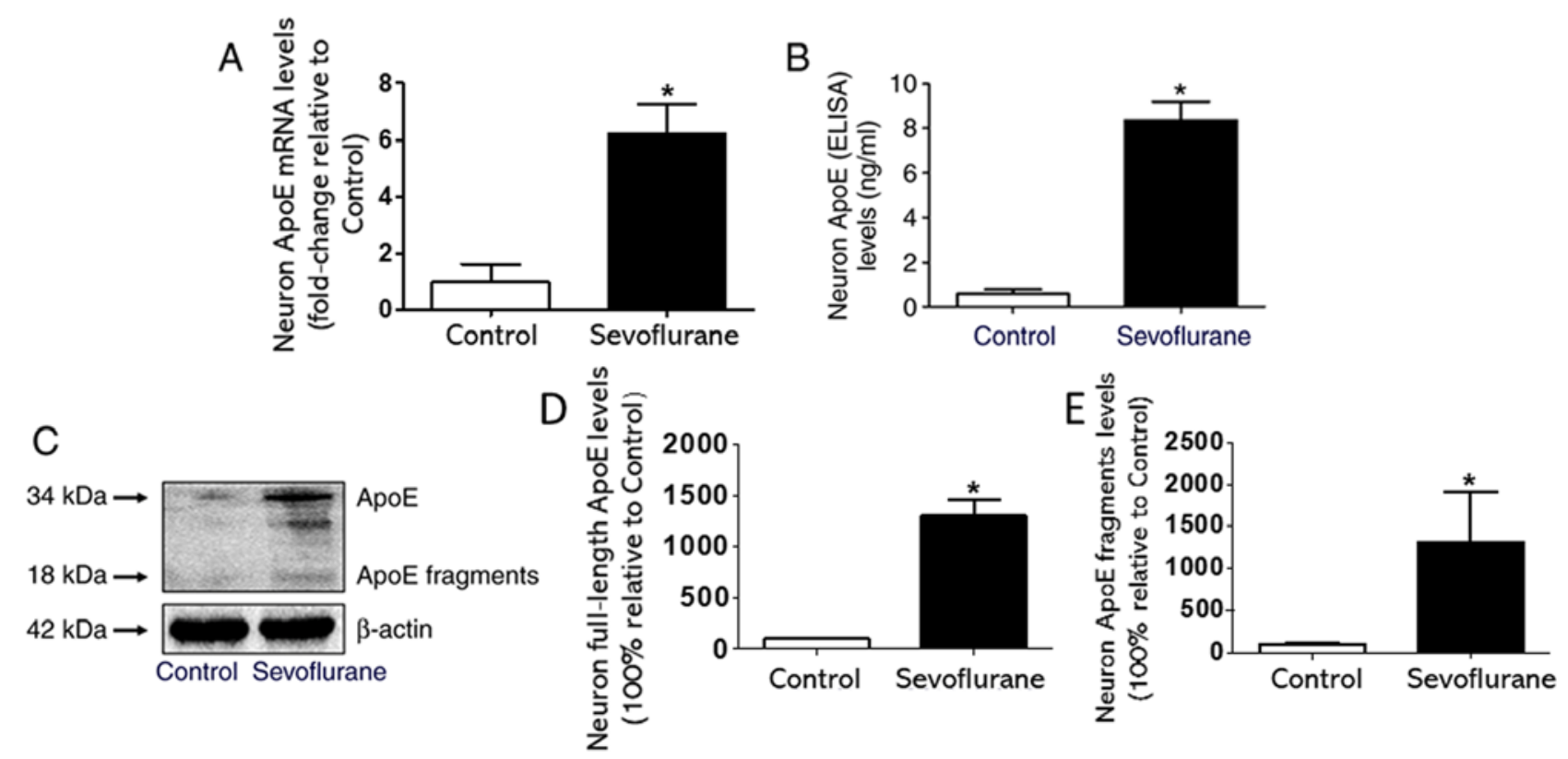

Figure 2. Effects of sevoflurane anesthesia on ApoE expression in mouse hippocampal neurons. Neurons were collected immediately after sevoflurane or control treatment to extract protein and RNA. (A) Reverse transcription-quantitative PCR was used to compare the expression of ApoE mRNA in neurons between different groups. (B) ELISA was used to compare the total ApoE content of neurons between the two groups. (C-E) Western blotting was used to compare the contents of (D) full-length ApoE and (E) ApoE toxic fragments in neurons between the control and sevoflurane groups. $n=4$ per group. ${ }^{*} \mathrm{P}<0.05$. ApoE, apolipoprotein E.

the control group, the expression levels of full-length ApoE and its $18-\mathrm{kDa}$ toxic fragments increased significantly in the sevoflurane group (Fig. 2C-E). In addition, compared with the neurons in the control group, the neurons treated with $4.1 \%$ sevoflurane for $4 \mathrm{~h}$ expressed higher levels of ApoE mRNA (Fig. 2A). The ELISA results indicated that the sevoflurane group expressed higher levels of total ApoE compared with the control group (Fig. 2B).

Sevoflurane anesthesia increases tau phosphorylation in mouse hippocampal neurons. The levels of total tau (Tau5) and phosphorylated tau (AT8 and PHF1) in neurons were compared between the control and sevoflurane groups by western blotting. The results demonstrated that the ratios of AT8 to Tau5 and PHF1 to Tau5 increased significantly after sevoflurane treatment (Fig. 3A-C). The immunofluorescence results also demonstrated that the ratio of AT8-positive to MAP2-positive neurons in sevoflurane group was significantly higher compared with the control group (Fig. 3).

Sevoflurane anesthesia induces neuroinflammation in mouse hippocampal neurons. ELISA was used to detect the expression of inflammatory factors in neurons. The results demonstrated that the levels of TNF- $\alpha$, IL- 6 and IL- $1 \beta$ in primary neurons were increased in the sevoflurane group compared with the control group (Fig. 4).

CoQ10 alleviates sevoflurane anesthesia-induced ATP, SOD and cell viability decrease in mouse hippocampal neurons. The contents of ATP and SOD were significantly decreased in the sevoflurane+corn oil group neurons compared with the control+corn oil group neurons (Fig. 5B and C). In addition, cell viability in the sevoflurane+corn oil group neurons was reduced compared with the control+corn oil group neurons (Fig. 5A). However, no significant differences were observed in ATP levels, SOD expression or cell viability between neurons in the sevoflurane+CoQ10 and the control+CoQ10 groups (Fig. 5).

CoQ10 mitigates sevoflurane-induced ApoE expression in mouse hippocampal neurons. The RT-qPCR and ELISA results demonstrated that ApoE gene and protein levels were significantly higher in the sevoflurane+corn oil group compared with those in the control+corn oil group (Fig. 6A and B). In addition, the western blotting results indicated that the expression of full-length ApoE and its toxic fragments were increased in the sevoflurane+corn oil group compared with the control+corn oil group (Fig. 6C-E). However, after CoQ10 treatment, no significant differences were observed in ApoE protein or mRNA levels between the control and sevoflurane groups (Fig. 6).

CoQ10 decreases sevoflurane-induced tau phosphorylation in mouse hippocampal neurons. According to the western blotting results, no significant differences were observed in the expression level of Tau 5 between the control+corn oil and the sevoflurane+corn oil groups, whereas the ratios of AT8 to Tau5 and PHF1 to Tau5 increased significantly after sevoflurane treatment compared with the control group. The immunofluorescence results demonstrated that the ratio of AT8-positive to MAP2-positive neurons in sevoflurane+corn oil group was significantly higher compared with the control+corn oil group. (Fig. 7D and E). No significant differences were observed in the above indices between the control+CoQ10 group and the sevoflurane+CoQ10 group (Fig. 7). 
A

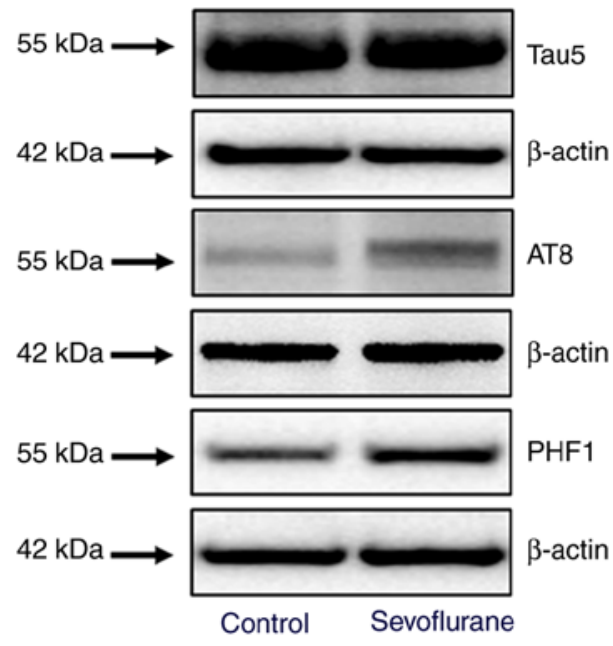

D

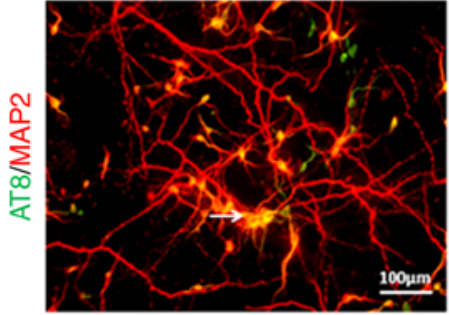

Control

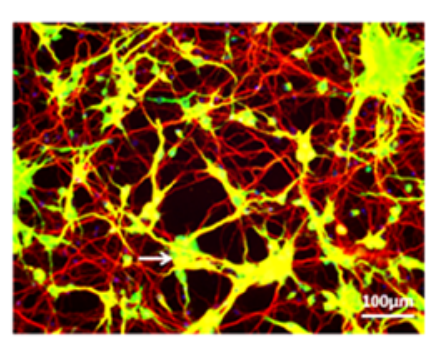

Sevoflurane
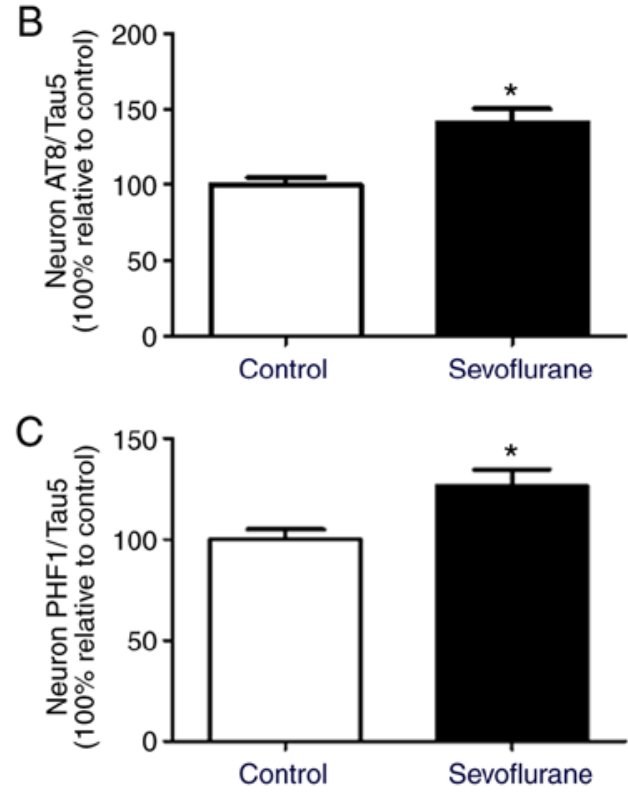

E

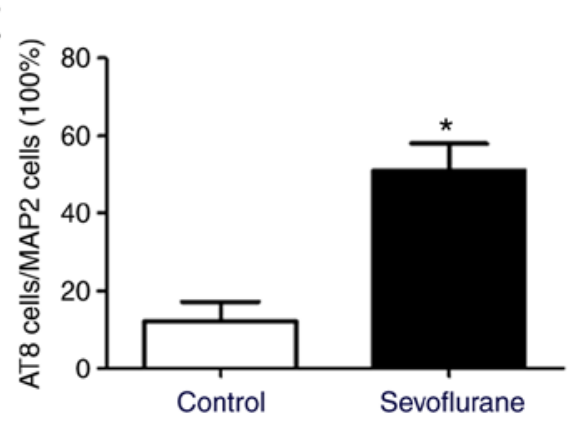

Figure 3. Effects of sevoflurane anesthesia on tau phosphorylation in mouse hippocampal neurons. (A) Western blotting was used to compare the expression levels of Tau5, AT8 and PHF1 in neurons between the control and sevoflurane groups. (B and C) The western blotting results of AT8 and PHF1 expression were represented as the ratios of (B) AT8 to Tau5 and (C) PHF1 to Tau5. (D) Immunofluorescence was used to test the expression of AT8 in neurons of both groups. (E) AT8 expression in immunofluorescence staining was calculated as the ratio of AT8-positive neurons (green) to MAP2-positive neurons (red) in the stained area. $n=6$ in western blotting and $n=3$ in immunofluorescence per group. ${ }^{*} \mathrm{P}<0.05$. Tau5, total tau; AT8, Tau-PS202/PT205; PHF1, Tau-PSer396/404; MAP2, recombinant microtubule associated protein 2.
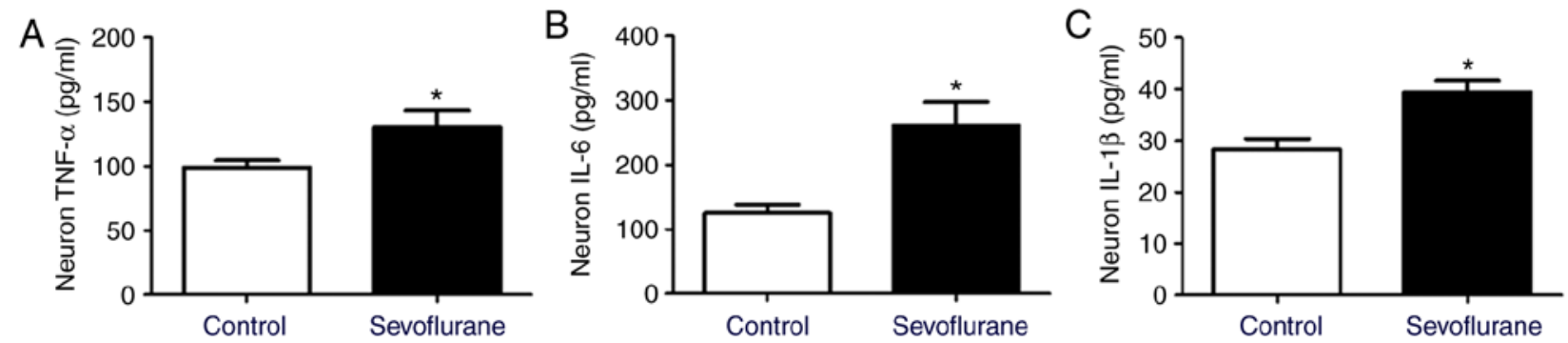

Figure 4. Effects of sevoflurane anesthesia on inflammatory cytokine levels in mouse hippocampal neurons. (A-C) Neurons were harvested following sevoflurane or control treatment for the evaluation of (A) TNF- $\alpha$, (B) IL-6 and (C) IL-1 $\beta$ levels. $n=4$ per group. *P<0.05 vs. control. TNF, tumor necrosis factor; IL, interleukin.

CoQ10 improves sevoflurane-induced neuroinflammation in mouse hippocampal neurons. The ELISA results demonstrated that the levels of TNF- $\alpha$, IL-6 and IL-1 $\beta$ were increased in the sevoflurane+corn oil group neurons compared with the control+corn oil group neurons (Fig. 8). After CoQ10 treatment, no significant differences were observed in inflammatory factor secretion between the control+CoQ10 and sevoflurane+CoQ10 groups (Fig. 8).

\section{Discussion}

Sevoflurane is the most commonly used inhalant anesthetic in pediatric surgery (1). However, studies have reported that repeated or long-term use of sevoflurane during induction and maintenance of general anesthesia in children may increase the risk of learning disability, thus greatly limiting the clinical application of sevoflurane $(19,20)$. 

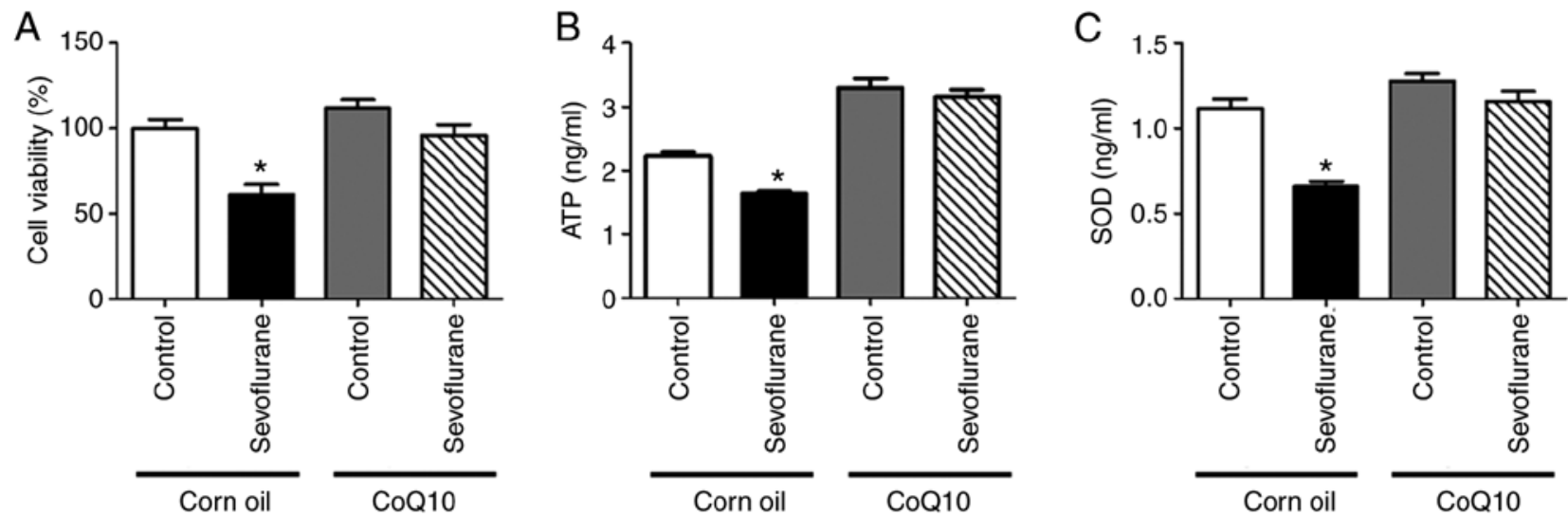

Figure 5. Effects of CoQ10 on cell viability, ATP levels and SOD contents in mouse hippocampal neurons following sevoflurane anesthesia. (A-C) Neurons were harvested after sevoflurane or control treatment; (A) CCK-8 assay was used to examine cell viability, and ELISA was used to measure the contents of (B) ATP and (C) SOD in neurons. $\mathrm{n}=4$ per group. "P<0.05 vs. control+corn oil. CoQ10, coenzyme Q10; SOD, superoxide dismutase.
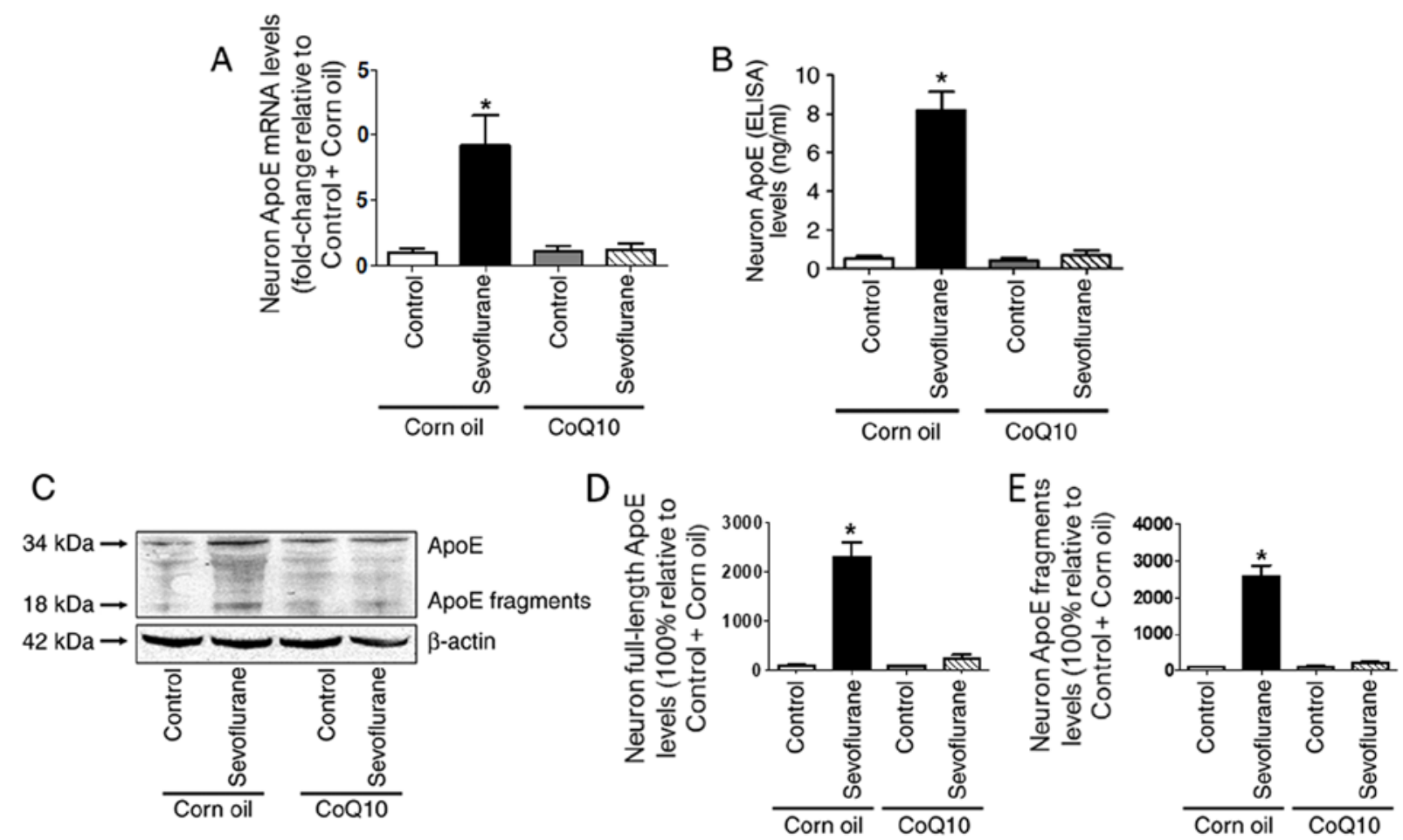

Figure 6. Effects of CoQ10 on ApoE levels in mouse hippocampal neurons following sevoflurane anesthesia. Neurons were collected immediately after sevoflurane or control treatment to extract protein and RNA. (A) Reverse transcription-quantitative PCR was used to compare the expression of ApoE mRNA in neurons among different groups. (B) ELISA was used to compare the total ApoE content of neurons among the groups. (C-E) Western blotting was used to compare the contents of (D) full-length ApoE and (E) ApoE toxic fragments in neurons among the control+corn oil, sevoflurane+corn oil, control+CoQ10 and sevoflurane + CoQ10 groups. $n=4$ per group. ${ }^{*} \mathrm{P}<0.05$ vs. control+corn oil. CoQ10, coenzyme Q10; ApoE, apolipoprotein E.

Lu et al (5) demonstrated that $4.1 \%$ sevoflurane exposure for $4 \mathrm{~h}$ affected the phosphorylation and neuroinflammatory activity of tau protein in primary neurons cultured for 5 days, but the specific mechanism remained unclear. In the present study, the cell model of $\mathrm{Lu}$ et al (5) was used to investigate the effects of high-concentration sevoflurane treatment on mouse hippocampal neurons. The results demonstrated that the contents of ATP and SOD, as well as cell viability decreased, whereas the levels of tau phosphorylation and inflammatory factors in the primary neurons were significantly increased after $4 \mathrm{~h}$ of sevoflurane treatment compared with those in the control group.
ApoE is a polymorphic protein involved in the transformation and metabolism of lipoproteins, and its gene can regulate multiple biological functions including lipid metabolism regulation and immune regulation (21). At present, the study of ApoE and its gene polymorphism is one of the hot topics in medical research (22). ApoE is considered to be a risk factor for AD (23). Generally, ApoE is expressed mainly by astrocytes in the brain, but in the case of injury or oxidative stress, neurons also activate the expression of ApoE to promote their repair $(24,25)$. When ApoE is hydrolyzed into neurotoxic protein fragments, they enter the cytoplasm, causing tau 

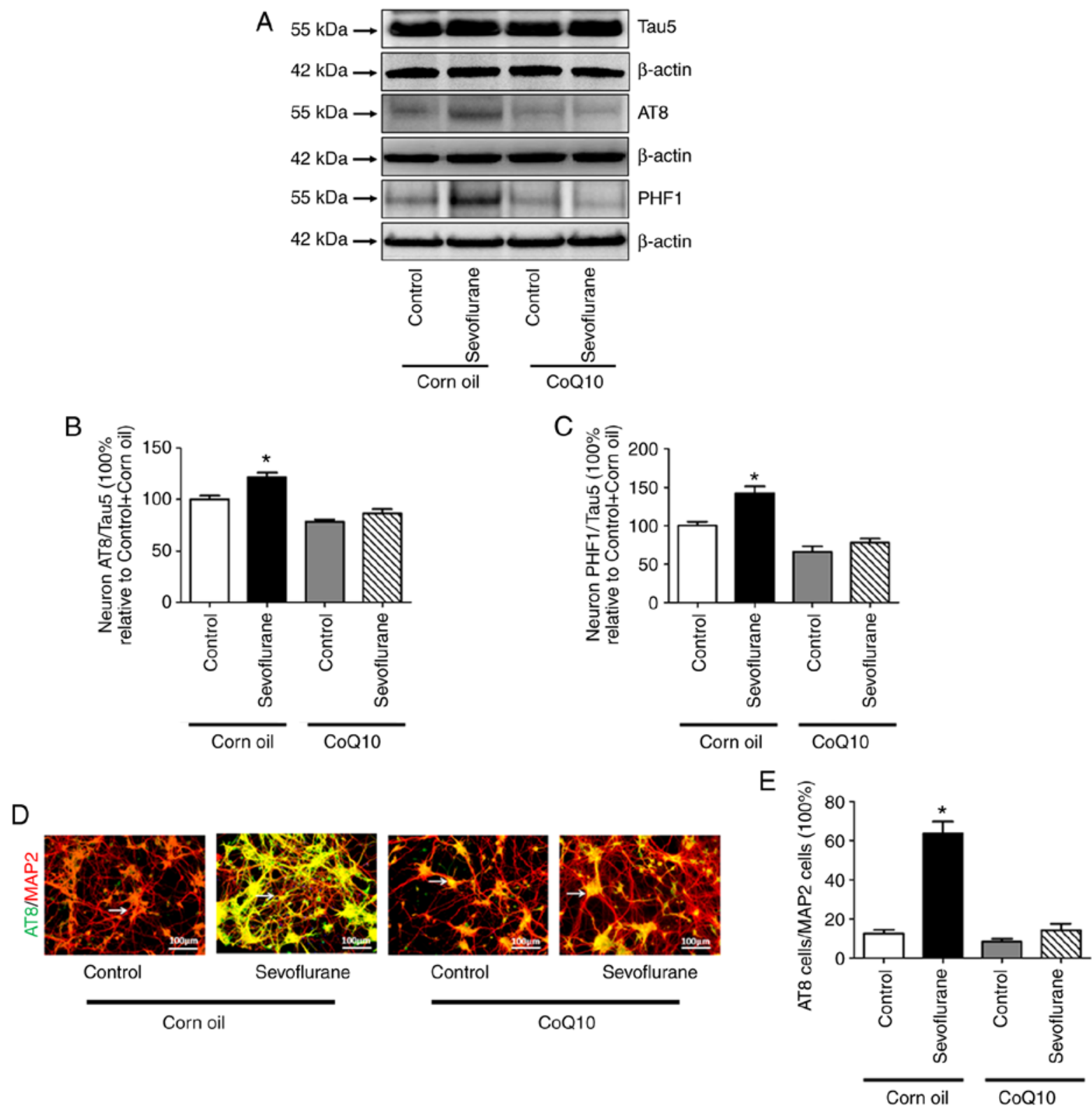

Figure 7. Effects of CoQ10 on tau phosphorylation in mouse hippocampal neurons following sevoflurane anesthesia. (A) Western blotting was used to measure the expression levels of Tau5, AT8 and PHF1 in neurons. (B and C) The western blotting results of AT8 and PHF1 expression were presented as the ratios of (B) AT8 to Tau5 and (C) PHF1 to Tau5. (D) Immunofluorescence assay was used to evaluate the expression of AT8 in the neurons of all the groups. (E) AT8 expression in immunofluorescence staining was calculated as the ratio of AT8-positive neurons (green) to MAP2-positive neurons (red) in the stained area. $n=6$ in western blotting and $n=3$ in immunofluorescence per group). "P<0.05 vs. control+corn oil. CoQ10, coenzyme Q10; Tau5, total tau; AT8, Tau-PS202/PT205; PHF1, Tau-PSer396/404; MAP2, recombinant microtubule associated protein 2.
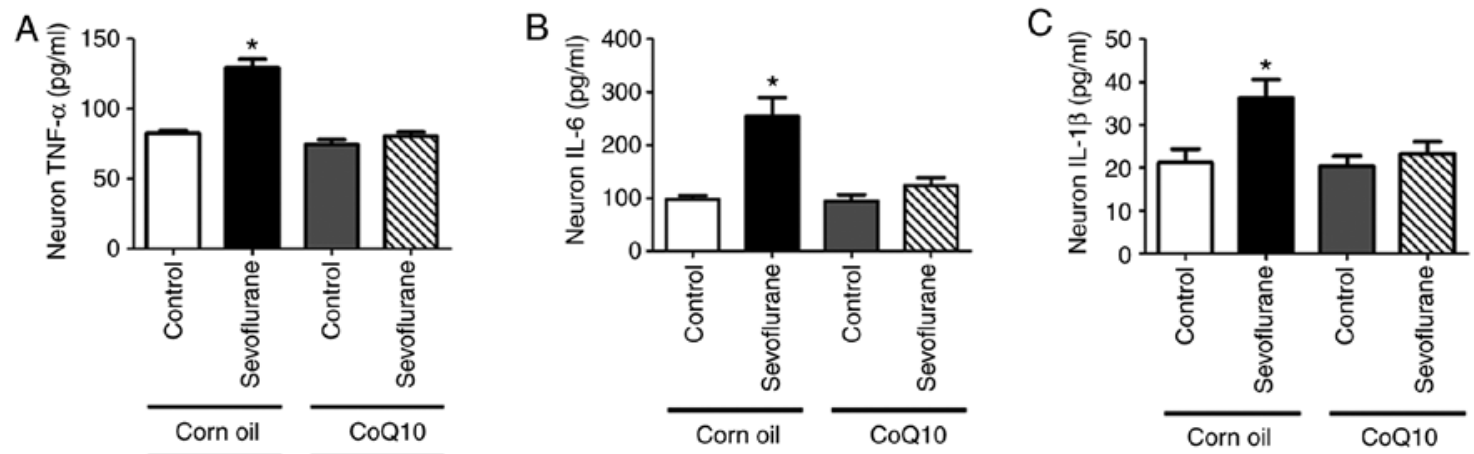

Figure 8. Effects of sevoflurane on inflammatory cytokine levels in mouse hippocampal neurons following sevoflurane anesthesia. (A-C) Neurons were harvested after sevoflurane or control treatment for the determination of (A) TNF- $\alpha$, (B) IL-6 and (C) IL-1 $\beta$ levels. $n=4$ per group. ${ }^{*}<0.05$ vs. control+corn oil. TNF, tumor necrosis factor; IL, interleukin. 
protein lesions and mitochondrial damage, further leading to neurodegeneration (12). Since $\gamma$-aminobutyric acid neurons are mainly located in the hippocampus and are responsible for cognitive functions such as learning and memory, which is particularly sensitive to the toxicity of ApoE fragments, the early symptoms of AD include cognitive impairment (13). A previous study has observed that repeated inhalation of sevoflurane can lead to oxidative stress in the developing brain, thus affecting cognitive functions (6). It was also observed that repeated anesthesia with $2.6 \%$ sevoflurane not only reduced the volume of the hippocampus, but also increased the expression of the ApoE gene in the CA1 and CA 3 regions of the hippocampus (26). In the present study, western blotting, ELISA and RT-qPCR indicated that immature neurons expressed a small amount of ApoE and its fragments in the control group, whereas ApoE and its fragment expression significantly increased following treatment with sevoflurane. In addition, sevoflurane treatment decreased the ATP and SOD levels, but increased the phosphorylated tau (AT8 and PHF1) and inflammatory factor (TNF- $\alpha$, IL-6 and IL-1 $\beta$ ) levels compared with those in the control group. These data indicated that sevoflurane treatment not only caused energy deficiency and oxidative stress, but also led to tau hyperphosphorylation and neuroinflammation; all of these processes may be associated with the enhancement of ApoE expression.

CoQ10 is an endogenous lipid-soluble antioxidant that is mainly present in the mitochondrial membrane (16). CoQ10 is an important cofactor in the mitochondrial electron transport chain; it exerts neuroprotective, energy conversion, anti-inflammatory and antioxidative effects (15). In particular, CoQ10 can improve bioenergy metabolism and mitochondrial function, increase brain energy level and improve the brain function of rodents (27). A preliminary experiment confirmed that the treatment dose of $500 \mu \mathrm{g} /$ well reversed the sevoflurane neurotoxicity (data not shown). Therefore, $500 \mu \mathrm{g} /$ well was chosen as the treatment. In the present study, hippocampal neurons were pre-treated with $1.5 \mathrm{mg} / \mathrm{ml} \mathrm{CoQ} 10$ (dissolved in corn oil) for $4 \mathrm{~h}$ before inhalation of oxygen and sevoflurane in the sevoflurane+CoQ10 and Control+CoQ10 groups; the results demonstrated that sevoflurane significantly increased ApoE, ApoE fragment and phosphorylated tau levels, and decreased the ATP and SOD levels in the sevoflurane+corn oil group, but not in the sevoflurane+CoQ10 group. These results suggested that CoQ10 increased the ATP level, but decreased the oxidative stress level of neurons, thus alleviating the high levels of ApoE and ApoE fragments produced by sevoflurane stimulation and mitigating the neuroinflammation caused by the sevoflurane-induced tau hyperphosphorylation in the neurons.

However, the present study has several limitations. Firstly, it was confirmed that the primary neurons cultured for 5 days were in their infancy; however, whether these neurons accurately represented the 6 day-old mouse sevoflurane anesthesia model was uncertain. Secondly, the role of ApoE in the neurons of ApoE-knockdown or -knockout mice was not addressed. Lastly, only ELISA was used to detect the inflammation-related indicators, such as TNF- $\alpha$, IL- 6 and IL-1 $\beta$; the protein levels of these indicators should be assessed by western blotting.
In summary, the present study demonstrated that sevoflurane treatment impaired mouse hippocampal neurons, which may be associated with expression of ApoE and its toxic fragments. CoQ10 improved energy replenishment and inhibited oxidative stress, which may lead to a decrease in ApoE and phosphorylated tau protein expression, thus mitigating the sevoflurane-induced neuroinflammation in mouse hippocampal neurons.

\section{Acknowledgements}

Not applicable.

\section{Funding}

This study was supported by the Tianjin Natural Science Foundation (grant no. 18JCZDJC35100) and the Science and Technology Development Fund of Tianjin Education Commission for Higher Education (grant no. 2017KJ194).

\section{Availability of data and materials}

The datasets used and/or analyzed during the current study are available from the corresponding author on reasonable request.

\section{Authors' contributions}

MY, YoY and YaY conceived and designed the study. MY, KX NL, YW and YaY performed the experiments. MY and YaY wrote the manuscript. KX and YoY reviewed and edited the manuscript. All authors read and approved the final manuscript.

\section{Ethics approval and consent to participate}

All experimental protocols in the present study were approved by the Animal Experimental Ethics Committee of Tianjin Medical University General Hospital (Tianjin, China; approval no. 2018-X6-11).

\section{Patient consent for publication}

Not applicable.

\section{Competing interests}

The authors declare that they have no competing interests.

\section{References}

1. Flick RP, Katusic SK, Colligan RC, Wilder RT, Voigt RG, Olson MD, Sprung J, Weaver AL, Schroeder DR and Warner DO: Cognitive and behavioral outcomes after early exposure to anesthesia and surgery. Pediatrics 128: e1053-e1061, 2011.

2. Zhou R, Li X, Li L and Zhang H: Theaflavins alleviate sevoflurane-induced neurocytotoxicity via Nrf2 signaling pathway. Int J Neurosci 130: 1-8, 2020.

3. Wilder RT, Flick RP, Sprung J, Katusic SK, Barbaresi WJ, Mickelson C, Gleich SJ, Schroeder DR, Weaver AL and Warner DO: Early exposure to anesthesia and learning disabilities in a population-based birth cohort. Anesthesiology 110: 796-804, 2009.

4. Raper J, Alvarado MC, Murphy KL and Baxter MG: Multiple anesthetic exposure in infant monkeys alters emotional reactivity to an acute stressor. Anesthesiology 123: 1084-1092, 2015. 
5. Lu H, Liufu N, Dong Y, Xu G, Zhang Y, Shu L, Soriano SG, Zheng H, Yu B and Xie Z: Sevoflurane acts on ubiquitination-proteasome pathway to reduce postsynaptic density 95 protein levels in young mice. Anesthesiology 127: 961-975, 2017.

6. Tao G, Zhang J, Zhang L, Dong Y, Yu B, Crosby G, Culley DJ, Zhang Y and Xie Z: Sevoflurane induces tau phosphorylation and glycogen synthase kinase $3 \beta$ activation in young mice. Anesthesiology 121: 510-527, 2014.

7. Zheng H, Dong Y, Xu Z, Crosby G, Culley DJ, Zhang Y and Xie Z Sevoflurane anesthesia in pregnant mice induces neurotoxicity in fetal and offspring mice. Anesthesiology 118: 516-526, 2013.

8. Zhou H, Li S and Wang G: Euxanthone ameliorates sevoflurane-induced neurotoxicity in neonatal mice. J Mol Neurosci 68: 275-286, 2019.

9. DiMaggio C, Sun LS, Kakavouli A, Byrne MW and Li G: A retrospective cohort study of the association of anesthesia and hernia repair surgery with behavioral and developmental disorders in young children. J Neurosurg Anesthesiol 21: 286-291, 2009.

10. Fuentes D, Fernández N, García Y, García T, Morales AR and Menéndez R: Age-related changes in the behavior of apolipoprotein E knockout mice. Behav Sci (Basel) 8: E33, 2018.

11. Rohn TT, Catlin LW, Coonse KG and Habig JW: Identification of an amino-terminal fragment of apolipoprotein E4 that localizes to neurofibrillary tangles of the Alzheimer's disease brain. Brain Res 1475: 106-115, 2012.

12. Munoz SS, Garner B and Ooi L: Understanding the role of ApoE fragments in Alzheimer's disease. Neurochem Res 44 1297-1305, 2019.

13. Wang C, Najm R, Xu Q, Jeong DE, Walker D, Balestra ME, Yoon SY, Yuan H, Li G, Miller ZA, et al: Gain of toxic apolipoprotein E4 effects in human iPSC-derived neurons is ameliorated by a small-molecule structure corrector. Nat Med 24: 647-657, 2018.

14. Shen X, Dong Y, Xu Z, Wang H, Miao C, Soriano SG, Sun D, Baxter MG, Zhang Y and Xie Z: Selective anesthesia-induced neuroinflammation in developing mouse brain and cognitive impairment. Anesthesiology 118: 502-515, 2013.

15. Turunen M, Olsson J and Dallner G: Metabolism and function of coenzyme Q. Biochim Biophys Acta 1660: 171-199, 2004.

16. Stefely JA and Pagliarini DJ: Biochemistry of mitochondrial coenzyme Q biosynthesis. Trends Biochem Sci 42: 824-843, 2017.

17. Xu G, Lu H, Dong Y, Shapoval D, Soriano SG, Liu X, Zhang Y and Xie Z: Coenzyme Q10 reduces sevoflurane-induced cognitive deficiency in young mice. Br J Anaesth 119: 481-491, 2017.
18. Livak KJ and Schmittgen TD: Analysis of relative gene expression data using real-time quantitative PCR and the 2(-Delta Delta C(T)) method. Methods 25: 402-408, 2001.

19. Lee JR and Loepke AW: Does pediatric anesthesia cause brain damage?-Addressing parental and provider concerns in light of compelling animal studies and seemingly ambivalent human data. Korean J Anesthesiol 71: 255-273, 2018

20. Sun L: Early childhood general anaesthesia exposure and neurocognitive development. Br J Anaesth 105 (Suppl 1): i61-i68, 2010.

21. Elliott DA, Tsoi K, Holinkova S, Chan SL, Kim WS, Halliday GM, Rye KA and Garner B: Isoform-specific proteolysis of apolipoprotein-E in the brain. Neurobiol Aging 32: 257-271, 2011.

22. van der Kant R, Goldstein LSB and Ossenkoppele R Amyloid- $\beta$-independent regulators of tau pathology in Alzheimer disease. Nat Rev Neurosci 21: 21-35,2020.

23. Dai J, Johnson ECB, Dammer EB, Duong DM, Gearing M, Lah JJ, Levey AI, Wingo TS and Seyfried NT: Effects of APOE genotype on brain proteomic network and cell type changes in Alzheimer's disease. Front Mol Neurosci 11: 454, 2018.

24. Brecht WJ, Harris FM, Chang S, Tesseur I, Yu GQ, Xu Q, Dee Fish J, Wyss-Coray T, Buttini M, Mucke L, et al: Neuron-specific apolipoprotein e4 proteolysis is associated with increased tau phosphorylation in brains of transgenic mice. J Neurosci 24: 2527-2534, 2004.

25. Morrow JA, Segall ML, Lund-Katz S, Phillips MC, Knapp M, Rupp B and Weisgraber KH: Differences in stability among the human apolipoprotein $\mathrm{E}$ isoforms determined by the amino-terminal domain. Biochemistry 39: 11657-11666, 2000.

26. Jiang J, Tang C, Ren J, Zhang C, Dong L and Zhu Z: Effect of multiple neonatal sevoflurane exposures on hippocampal apolipoprotein E levels and learning and memory abilities. Pediatr Neonatol 59: 154-160, 2018

27. Mohammadi-Bardbori A, Najibi A, Amirzadegan N, Gharibi R, Dashti A, Omidi M, Saeedi A, Ghafarian-Bahreman A and Niknahad H: Coenzyme Q10 remarkably improves the bio-energetic function of rat liver mitochondria treated with statins. Eur J Pharmacol 762: 270-274, 2015.

This work is licensed under a Creative Commons Attribution-NonCommercial-NoDerivatives 4.0 International (CC BY-NC-ND 4.0) License. 